\title{
Holographic model of hybrid and coexisting s-wave and p-wave Josephson junction
}

\author{
Shuai Liu ${ }^{\mathrm{a}}$, Yong-Qiang Wang ${ }^{\mathrm{b}}$ \\ Institute of Theoretical Physics, Lanzhou University, Lanzhou 730000, People's Republic of China
}

Received: 15 August 2015 / Accepted: 19 September 2015 / Published online: 16 October 2015

(C) The Author(s) 2015. This article is published with open access at Springerlink.com

\begin{abstract}
In this paper the holographic model for a hybrid and coexisting s-wave and p-wave Josephson junction is constructed by a triplet charged scalar field coupled with a nonAbelian $S U$ (2) gauge field in (3+1)-dimensional AdS spacetime. Depending on the value of chemical potential $\mu$, one can show that there are four types of junctions $(s+p-N-s+p$, $s+p-N-s, s+p-N-p$ and $s-N-p)$. We show that the DC currents of all the hybrid and coexisting s-wave and p-wave junctions are proportional to the sine of the phase difference across the junction. In addition, the maximum current and the total condensation decay with the width of junction exponentially, respectively. For the $\mathrm{s}+\mathrm{p}-\mathrm{N}-\mathrm{s}$ and $\mathrm{s}-\mathrm{N}-\mathrm{p}$ junctions, the maximum current decreases with growing temperature. Moreover, we find that the maximum current increases with growing temperature for the $s+p-N-s+p$ and $s+p-N-p$ junctions, which is different from the behavior of the $s+p-N-s$ and $s-N-p$ junctions.
\end{abstract}

\section{Contents}

1 Introduction . . . . . . . . . . . . . 1

2 Holographic model of hybrid and coexisting s-wave and p-wave Josephson junction . . . . . . . . . 2

2.1 The model setup . . . . . . . . . . . . 2

2.2 The scaling symmetry .......... . . . . . 4

3 Numerical results ............... . . 4

$3.1 \mathrm{~s}+\mathrm{p}-\mathrm{N}-\mathrm{s}+\mathrm{p}$ Josephson junction ... . . . . . 5

$3.2 \mathrm{~s}+\mathrm{p}-\mathrm{N}-\mathrm{s}$ Josephson junction . . . . . . . . . . 6

$3.3 \mathrm{~s}+\mathrm{p}-\mathrm{N}-\mathrm{p}$ Josephson junction . . . . . . . . . 7

3.4 s-N-p Josephson junction . . . . . . . . . . . 9

4 Conclusion and discussion . . . . . . . . . . . . 10

References ............... 11

\footnotetext{
a e-mail: liush13@1zu.edu.cn

be-mail: yqwang@lzu.edu.cn
}

\section{Introduction}

The study of superconductivity has been at the forefront of condensed matter physics. In particular, what the origin is of high temperature superconductivity is still one of the major unsolved problems of condensed matter theory. Over the past decade, one of the most important results in string theory is the $\mathrm{AdS} / \mathrm{CFT}$ correspondence, which was first proposed by Maldacena in $[1,2]$ and states that the strong coupled field living on the AdS boundary can be described with a weakly gravity theory in one higher-dimensional AdS spacetime. By applying the AdS/CFT correspondence, one has first achieved success in the study of holographic QCD and heavy ions collisions. In recent times, the AdS/CFT correspondence also has provided insights into condensed matter theory. In [3-5], the authors investigated the action of a complex scalar field coupled to a $U(1)$ gauge field in a $(3+1)$-dimensional Schwarzschild-AdS black hole and found that below some critical value of the temperature due to the $U(1)$ symmetry breaking, the scalar field which condenses near the horizon could be interpreted as a Cooper pair-like superconductor condensation. Moreover, analyzing the optical conductivity of the superconducting state, the rate of the width of the gap to the critical temperature is close to the value of a high temperature superconductor. Thus, it is hoped that the holographic model can match the properties of the high temperature superconducting behavior. Soon, The p-wave and d-wave holographic superconductors proposed in [6-8], respectively. For reviews of holographic superconductors, see [9-12].

It is well known that the Josephson junction is a device made up of two superconductor materials coupled by weak link barrier in [13]. The weak link can be a thin normal conductor; then it is named a superconductor-normalsuperconductor junction (SNS), or a thin insulating barrier; then we have a superconductor-insulator-superconductor junction (SIS). Recently, by studying the space-dependent solution of the action of a Maxwell field coupled with a com- 
plex scalar field in a $(3+1)$-dimensional Schwarzschild-AdS black hole background, Horowitz et al. [14] had constructed a $(2+1)$-dimensional holographic model of a Josephson junction and found a sine relation between the tuning current and the phase difference of the condensation across the junction. The extension to a 4-dimensional Josephson junction has been discussed in $[15,16]$. The holographic mode of a Josephson junction array has been constructed based on a designer multigravity in [17]. With the $S U(2)$ gauge field coupled with gravity, the holographic p-wave Josephson junction has also been discussed in [18]. In [19], a holographic model of a $(1+1)$-dimensional superconductor-insulatorsuperconductor (S-I-S) Josephson junction has been investigated. In $[20,21]$, with the action of the Einstein-Maxwellcomplex scalar field, the authors studied a holographic model of superconducting quantum interference device (SQUID). In [22] a holographic model of a Josephson junction in the non-relativistic case with a Lifshitz geometry was constructed.

Recently, the holographic approach has been applied to the coexistence and competition order phenomena, in which the phase diagram has a rich structure, such as the competition of two scalar order parameters in the probe limit $[23,24]$ and with the backreaction of the scalars [25], the coexistence of two p-wave order parameters [26], and the competition of s-wave and d-wave order parameters [27]. Especially, there is another interesting paper about competition and coexistence of s-wave and p-wave order in $[28,29]$; the authors studied a charged triplet scalar coupled with an $S U(2)$ gauge field in the $(3+1)$-dimensional spacetime and confirmed $\mathrm{s}+\mathrm{p}$ coexisting phases. Furthermore, the phase transitions of the holographic $\mathrm{s}+\mathrm{p}$-wave superconductor with backreaction is investigated in Ref. [30].

Since the holographic model of hybrid and $\mathrm{s}+\mathrm{p}$ coexisting superconductors has been constructed, it is natural to set up a holographic model for hybrid and coexisting s-wave and p-wave Josephson junction. We will study a non-Abelian $S U$ (2) Yang-Mills field and a scalar triplet charged under an $S U$ (2) gauge field in (3+1)-dimensional AdS spacetime and construct the holographic model of a hybrid and coexisting s-wave and p-wave Josephson junction, which will be related to the s-N-s junction in [14] and the p-N-p junction in [18]. To construct the holographic model of a junction, ones need to tune the value of the chemical potential.

The paper is organized as follows: in Sect. 2, we consider a non-Abelian $S U$ (2) gauge field coupled with a scalar triplet charged field in $(3+1)$-dimensional AdS spacetime and construct a gravity dual model for a $(2+1)$-dimensional $s+p$ coexisting Josephson junction. We show the numerical results of the equations of motion and study the characteristics of the $(2+1)$-dimensional $s+p$ coexisting Josephson junction in Sect. 3. The conclusion and discussion are in the last section.

\section{Holographic model of hybrid and coexisting s-wave and p-wave Josephson junction}

\subsection{The model setup}

In [28], the author considered the action of a charged scalar field coupled to an $S U$ (2) gauge field, in which the charged scalar field transform as a triplet under the gauge group $S U$ (2). This model can realize the competition and coexistence of s-wave and p-wave order parameters. We will also adopt the same form of the action in the $(3+1)$-dimensional AdS spacetime:

$$
\begin{aligned}
S= & \frac{1}{2 \kappa_{g}^{2}} \int \mathrm{d}^{4} x \sqrt{-g}(R-2 \Lambda) \\
& +\frac{1}{g_{c}^{2}} \int \mathrm{d}^{4} x \sqrt{-g}\left(-\left|D_{\mu} \Psi^{a}\right|^{2}-\frac{1}{4} F_{\mu \nu}^{a} F^{a \mu \nu}-m^{2}\left|\Psi^{a}\right|^{2}\right),
\end{aligned}
$$

where $\Lambda=-3 / L^{2}$ is the negative cosmology constant, and $L$ is the radius of asymptotic AdS spacetime. $\Psi^{a}$ is a complex scalar triplet charged under the $S U$ (2) Yang-Mills field and

$D_{\mu} \Psi^{a}=\partial_{\mu} \Psi^{a}+\varepsilon^{a b c} A_{\mu}^{b} \Psi^{c}$.

The field strength $F_{\mu \nu}^{a}$ of the $S U(2)$ gauge theory is given by

$F_{\mu \nu}^{a}=\partial_{\mu} A_{\nu}^{a}-\partial_{\nu} A_{\mu}^{a}+\varepsilon^{a b c} A_{\mu}^{b} A_{\nu}^{c}$,

where the one form $A=A_{\mu} \mathrm{d} x^{\mu}=A_{\mu}^{a} \tau^{a} \mathrm{~d} x^{\mu}$ and the superscript a is the index of generator $\tau^{a}$ of $S U$ (2) gauge field with $a=1,2,3 . g_{\mathrm{c}}$ is the coupling constant of non-Abelian $S U$ (2) Yang-Mills field. In this paper we will consider the probe limit by taking $g_{\mathrm{c}} \rightarrow \infty$ to ignore the backreaction of the matter.

In the probe limit, we still choose a $(3+1)$-dimensional planar Schwarzschild-AdS black hole solution as the background geometry with metric

$\mathrm{d} s^{2}=-f(r) \mathrm{d} t^{2}+\frac{\mathrm{d} r^{2}}{f(r)}+r^{2}\left(\mathrm{~d} x^{2}+\mathrm{d} y^{2}\right)$,

where $x$ and $y$ are the coordinates of a 2-dimensional Euclidean space. The function $f(r)$ is

$f(r)=\frac{r^{2}}{L^{2}}\left(1-\frac{r_{h}^{3}}{r^{3}}\right)$,

where $r_{h}$ is the radius of the black hole's event horizon. The Hawking temperature of the black hole is given by

$$
T=\left.\frac{1}{4 \pi} \frac{\mathrm{d} f}{\mathrm{~d} r}\right|_{r=r_{h}}=\frac{3 r_{h}}{4 \pi L^{2}} .
$$


The temperature $T$ relates to the radius of the black hole's event horizon $r_{h}$ and the AdS radius $L$. In the rest of paper, we will work in unit in which $L=1 . T$ corresponds to the temperature of the dual field theory on the AdS boundary.

The variation of the action (2.1) with respect to the scalar field $\Psi^{a}$ and $A_{\mu}^{a}$ lead to the equations of motion, respectively. We have

$$
\begin{aligned}
& \nabla_{\mu}\left(D^{\mu} \Psi^{a}\right)+\varepsilon^{a b c} A_{\mu}^{b} D^{\mu} \Psi^{c}-m^{2} \Psi^{a}=0 \\
& \nabla^{v} F_{v \mu}^{a}+\varepsilon^{a b c} A^{b v} F_{v \mu}^{c}-2 \varepsilon^{a b c} \Psi^{b}\left(\partial_{\mu} \Psi^{c}+\varepsilon^{c d e} A_{\mu}^{d} \Psi^{e}\right)=0
\end{aligned}
$$

For the hybrid and coexisting s-wave and p-wave Josephson junction, there are several different kinds of matter fields ansatzes. Let us consider one of them:

$$
\begin{aligned}
& \widetilde{\Psi}^{3}=\widetilde{\Psi}_{3}(r, y), \quad A_{t}^{1}=\phi(r, y), \quad A_{x}^{3}=\Psi_{x}(r, y), \\
& A_{r}^{1}=A_{r}(r, y), \quad A_{y}^{1}=A_{y}(r, y),
\end{aligned}
$$

where the field functions $\widetilde{\Psi}^{3}, \theta^{a}, \phi, \Psi_{x}, A_{r}$, and $A_{y}$ are dependent of the coordinates $r$ and $y$. Thus the holographic model of the Josephson junction would be along the $y$ direction. Without loss of generality, we will consider the $S U$ (2) gauge-invariant vector field $A_{\mu}^{a}$ and the scalar field $\widetilde{\Psi}^{a}$ :

$M_{\mu}^{a}=A_{\mu}^{a}-D_{\mu} \theta^{a}, \quad \Psi^{a}=\widetilde{\Psi}^{a}+\varepsilon^{a b c} \theta^{b} \widetilde{\Psi}^{c}$.

With the black hole background (2.4) and the above ansatz (2.9), the equations of the matter fields (2.7) and (2.8) can be written as

$$
\begin{aligned}
& \partial_{r}^{2} \Psi_{3}+\frac{1}{r^{2} f} \partial_{y}^{2} \Psi_{3} \\
& +\left(\frac{f^{\prime}}{f}+\frac{2}{r}\right) \partial_{r} \Psi_{3}+\left(-\frac{m^{2}}{f}+\frac{\phi^{2}}{f^{2}}-M_{r}^{2}-\frac{M_{y}^{2}}{r^{2} f}\right) \Psi_{3}=0 \\
& \partial_{r}^{2} \phi+\frac{1}{r^{2} f} \partial_{y}^{2} \phi+\frac{2}{r} \partial_{r} \phi-\frac{\phi \Psi_{x}^{2}}{r^{2} f}-\frac{2 \phi \Psi_{3}^{2}}{f}=0 \\
& \partial_{r}^{2} \Psi_{x}+\frac{1}{r^{2} f} \partial_{y}^{2} \Psi_{x}+\frac{f^{\prime}}{f} \partial_{r} \Psi_{x}+\left(\frac{\phi^{2}}{f^{2}}-M_{r}^{2}-\frac{M_{y}^{2}}{r^{2} f}\right) \Psi_{x}=0 \\
& \partial_{y}^{2} M_{r}-\partial_{r} \partial_{y} M_{y}-M_{r} \Psi_{x}^{2}-2 r^{2} M_{r} \Psi_{3}^{2}=0 \\
& \partial_{r}^{2} M_{y}-\partial_{r} \partial_{y} M_{r}+\frac{f^{\prime}}{f}\left(\partial_{r} M_{y}-\partial_{y} M_{r}\right) \\
& \quad-\frac{M_{y} \Psi_{x}^{2}}{r^{2} f}-\frac{2 \Psi_{3}^{2} M_{y}}{f}=0,
\end{aligned}
$$

$$
\begin{aligned}
& \partial_{r} M_{r}+\frac{1}{r^{2} f} \partial_{y} M_{y}+\frac{2}{\Psi_{3}}\left(M_{r} \partial_{r} \Psi_{3}+\frac{M_{y}}{r^{2} f} \partial_{y} \Psi_{3}\right) \\
& +\left(\frac{f^{\prime}}{f}+\frac{2}{r}\right) M_{r}=0 \\
& \partial_{r} M_{r}+\frac{1}{r^{2} f} \partial_{y} M_{y}+\frac{2}{\Psi_{x}}\left(M_{r} \partial_{r} \Psi_{x}+\frac{M_{y}}{r^{2} f} \partial_{y} \Psi_{x}\right) \\
& \quad+\frac{f^{\prime}}{f} M_{r}=0
\end{aligned}
$$

where a prime denotes the derivative with respect to $r$. In our paper, we will work with the case $m^{2} \geq-9 / 4$ in order to satisfy the BF bound [31]. Let us inspect Eqs. (2.11a)-(2.11g). If $\Psi_{x}, A_{r}$, and $A_{y}$ are turned off, $\Psi_{3}$ and $\phi$ are only dependent on $r$, and the remaining two equations will be the same as the equations of the s-wave holographic superconductivity. Similarly, if we turn off $\Psi_{3}, A_{r}$, and $A_{y}, \Psi_{x}$ and $\phi$ are only dependent on $r$, and the two remaining equations will be the equations which describe the p-wave condensation. Next, we will consider the Josephson junction. If $\Psi_{x}$ is only turned off, the remaining equations are the same equations as of the pure s-wave Josephson junction. In a similar way, if we only turn off $\Psi_{3}$, we will get the pure p-wave Josephson junction. So we can get the so-called $s+p$ coexisting phase Josephson junction under this ansatz (2.9). It is obvious that Eqs. (2.11a)-(2.11g) are coupled and nonlinear, so we need to solve them numerically instead of solving them analytically.

Near the AdS boundary $(r \rightarrow \infty)$, the matter fields take the asymptotic forms

$$
\begin{aligned}
& \Psi_{3}=\frac{\Psi_{3}^{(-)}(y)}{r^{\Delta_{-}}}+\frac{\Psi_{3}^{(+)}(y)}{r^{\Delta_{+}}}+\ldots, \\
& \phi=\mu(y)-\frac{\rho(y)}{r}+\mathcal{O}\left(\frac{1}{r^{2}}\right), \\
& \Psi_{x}=\Psi_{x}^{(-)}(y)+\frac{\Psi_{x}^{(+)}(y)}{r}+\mathcal{O}\left(\frac{1}{r^{3}}\right), \\
& M_{r}=\mathcal{O}\left(\frac{1}{r^{3}}\right), \\
& M_{y}=v(y)+\frac{J}{r}+\mathcal{O}\left(\frac{1}{r^{2}}\right) .
\end{aligned}
$$

Here, the dimensions of the operations $\Psi_{3}^{( \pm)}$are

$\Delta_{ \pm}=\frac{3 \pm \sqrt{9+4 m^{2}}}{2}$

According to the AdS/CFT dictionary, $\Psi_{3}^{( \pm)}$is considered as the source of the scalar operation of s-wave condensation, and $\Psi_{3}^{(\mp)}$ is the corresponding expectation value of the operator. Meanwhile, $\Psi_{x}^{( \pm)}$is the source of the vector operation of p-wave condensation, and $\Psi_{x}^{(\mp)}$ is the corresponding expectation value of the operator. $\mu(y), \rho(y), v(y)$, and $J$ are the 
chemical potential, charge density, velocity of the superfluid, and the constant current in the dual field, respectively [3239].

In order to solve Eqs. (2.11a)-(2.11g) numerically, we need to impose the boundary conditions on them. First, we impose the Dirichlet boundary conditions on $\Psi_{3}$ and $\Psi_{x}$ on the AdS boundary. In this paper, we set

$\Psi_{3}^{(-)}=0$.

So $\Psi_{3}^{(+)}$is the expectation value of s-wave scalar operator, $\Psi_{3}^{(+)}=\left\langle\mathcal{O}_{s}\right\rangle$. Similarly, we impose the Dirichlet boundary condition on $\Psi_{x}$ on the AdS boundary:

$\Psi_{x}^{(-)}=0$.

$\Psi_{x}^{(+)}$is the expectation value of the p-wave vector operator, $\Psi_{x}^{(+)}=\left\langle\mathcal{O}_{p}\right\rangle$. Second, we impose the Dirichlet boundary condition on $\phi$ at the horizon:

$\phi\left(r_{h}\right)=0$.

$\phi$ is the $t$ component of $A_{\mu}^{1}$, and $\phi\left(r_{h}\right)=0$ is to avoid the divergence of $g^{\mu v} A_{\mu}^{1} A_{v}^{1}$. In addition, the matter field functions are independent of $y$ at the spatial coordinate $y \rightarrow \pm \infty$. So, the boundary conditions of Eqs. (2.11a)-(2.11g) are determined by the value of $\mu$ and $J$. The phase difference $\gamma$ across the junction is $\gamma=\Delta \theta^{1}-\int A_{y} \mathrm{~d} y$. The phase difference $\Delta \theta^{1}$ can be eliminated under the $S U(2)$ gaugeinvariant, thus it is convenient to set

$\gamma=-\int_{-\infty}^{+\infty} A_{y} \mathrm{~d} y$

Now, we would like to introduce the critical temperature $T_{\mathrm{c}}$ of the Josephson junction, which is proportional to the chemical potential $\mu(+\infty)$ or $\mu(-\infty)$, and we set

$T_{\mathrm{c}}=\frac{3}{4 \pi} \frac{\mu(-\infty)}{\mu_{\mathrm{c}}}$

where $\mu_{\mathrm{c}} \approx 3.65$. In order to describe a hybrid and coexisting $\mathrm{s}$-wave and p-wave Josephson junction, we should construct a chemical potential which can make a phase transition occurring along the direction $y$ of the Josephson junction; thus the chemical potential $\mu(y)$ is dependent on the spatial coordinate $y$,

$$
\begin{aligned}
\mu(y)=\mu & \left\{1-\frac{1-\epsilon}{2 \tanh \left(\frac{\ell}{2 \sigma}\right)}\right. \\
\times & {\left.\left[\tanh \left(\frac{y+\frac{\ell}{2}}{\sigma}\right)-\beta \tanh \left(\frac{y-\frac{\ell}{2}}{\sigma}\right)\right]\right\}, }
\end{aligned}
$$

where the chemical potential $\mu(y)$ is proportional to $\mu$ and $\ell$ is the width of the Josephson junction. We use the parameter $\epsilon$, and $\sigma$ and $\beta$ control the steepness and the depth of the junction, respectively.

\subsection{The scaling symmetry}

Analyzing the EoM, we found that there is a scaling symmetry in Eqs. (2.11a)-(2.11g). These equations are invariant under the following scale transformation:

$$
\left\{\begin{array}{l}
r \rightarrow b r, \quad r_{h} \rightarrow b r_{h}, \quad y \rightarrow y / b, \quad \phi \rightarrow b \phi, \\
\Psi_{x} \rightarrow b \Psi_{x}, \quad \Psi_{3} \rightarrow \Psi_{3}, \quad f \rightarrow b^{2} f, \quad f^{\prime} \rightarrow b f^{\prime}, \quad A_{y} \rightarrow b A_{y} .
\end{array}\right.
$$

Under this scaling symmetry, we can get the behavior of the following physical quantities:

$\left\{\begin{array}{l}\mu \rightarrow b \mu, \quad \Psi_{3}^{(-)} \rightarrow b^{\Delta_{-}} \Psi_{3}^{(-)}, \quad \Psi_{3}^{(+)} \rightarrow b^{\Delta_{+}} \Psi_{3}^{(+)}, \\ \rho \rightarrow b^{2} \rho, \quad \Psi_{x}^{(-)} \rightarrow b \Psi_{x}^{(-)}, \quad \Psi_{x}^{(+)} \rightarrow b^{2} \Psi_{x}^{(+)}, \quad J \rightarrow b^{2} J .\end{array}\right.$

Because of this scaling symmetry, we can set the radius of the black hole's event horizon $r_{h}=1$. In addition, we have set $L=1$, so the temperature $T$ and the background geometry is fixed. From Eq. (2.6), we can see that the temperature $T$ changes to $b T$ under the scaling transformation. So the following quantities are invariant:

$\Psi_{3}^{(+)} / T^{\Delta_{+}}, \quad \Psi_{x}^{(+)} / T^{2}, \quad J / T^{2}$.

These invariant quantities can change with $T / T_{\mathrm{c}}$.

\section{Numerical results}

In this section we will solve the coupled and nonlinear equations (2.11a)-(2.11g) numerically. Before we solve these equations, we will have coordinate transformations, $z=1 / r$, $\tilde{y}=\tanh \left(\frac{y}{4 \sigma}\right)$. It is more convenient to impose boundary conditions at $z=1, z=0$, and $\tilde{y}= \pm 1$, rather than at $r=1$, $r=+\infty$, and $y= \pm \infty$. There are four kinds of Josephson junctions.

(i) The $\mathrm{s}+\mathrm{p}-\mathrm{N}-\mathrm{s}+\mathrm{p}$ Josephson junction consists of the $\mathrm{s}+\mathrm{p}$ coexisting phase in the two leads, and the normal phase between them.

(ii) The $\mathrm{s}+\mathrm{p}-\mathrm{N}-\mathrm{s}$ Josephson junction consists of the $\mathrm{s}+\mathrm{p}$ coexisting phase in the left lead, the conventional s-wave phase in the right lead, and the normal phase between them.

(iii) The $\mathrm{s}+\mathrm{p}-\mathrm{N}-\mathrm{s}$ Josephson junction consists of the $\mathrm{s}+\mathrm{p}$ coexisting phase in the left lead, the conventional p-wave phase in the right lead, and the normal phase between them. 
(iv) The s-N-p Josephson junction consists of the conventional s-wave phase in the left lead, the conventional $\mathrm{s}$-wave phase in the right lead, and the normal phase between them.

We can tune the chemical potential $\mu(y)$ to realize the four cases. In [28], when the value of $\Delta_{+}$or $m^{2}$ is in a special region, as the temperature decreases, the p-wave condensation will appear first and increase; the s-wave condensation will not appear. When the temperature continues to decrease and reaches the critical temperature $T_{c 1}^{s p}$, the s-wave condensation will appear and increase, and the $\mathrm{p}$-wave condensation will decrease. When the temperature reaches another critical temperature $T_{c 2}^{s p}$, the p-wave condensation will disappear. So when the temperature is in the region $T_{c 2}^{s p} \sim T_{c 1}^{s p}$, the swave phase and p-wave will coexist. When the temperature is higher than $T_{c 1}^{s p}$, there is only a p-wave phase. When the temperature is below $T_{c 2}^{s p}$, there is only an s-wave phase. In order to construct the junction of the $\mathrm{s}+\mathrm{p}$ coexisting phase, we can write the region of the chemical potential as $\mu_{c 1} \sim \mu_{c 2}$ in [28].

In this subsection, in order to obtain the model of a $\mathrm{s}+\mathrm{p}-\mathrm{N}-\mathrm{s}+\mathrm{p}$ Josephson junction, we need to tune the value of chemical potential $\mu(y)$ at $y= \pm \infty$ such that it is in the $\mathrm{s}+\mathrm{p}$ coexisting region $\mu_{c 1} \sim \mu_{c 2}$. Because the superconductor phase in the two leads are symmetrical, the phase difference $\gamma$ can be obtained by (2.21); we have

$\gamma=-\int_{-\infty}^{+\infty} \mathrm{d} y[v(y)-v( \pm \infty)]$

The profiles of $A_{t}^{1}$ and $A_{y}^{1}$ are shown in Fig. 1.

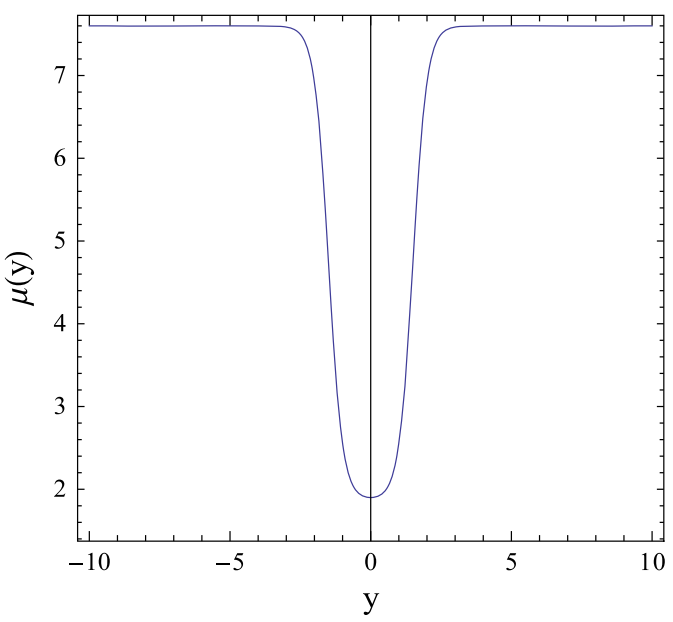

\section{$3.1 \mathrm{~s}+\mathrm{p}-\mathrm{N}-\mathrm{s}+\mathrm{p}$ Josephson junction}

We show the relationship between the DC current $J$ and the phase difference $\gamma$ across the junction on the left panel of Fig. 2. From the figure, we can see that $J$ is proportional to the sine of $\gamma$. We have

$J / T_{\mathrm{c}}^{2} \approx \begin{cases}0.464 \sin \gamma & \text { for } m^{2}=-33 / 16 \\ 0.539 \sin \gamma & \text { for } m^{2}=-1031 / 500\end{cases}$

Note that we only obtain the phase difference in the interval $(-\pi / 2, \pi / 2)$, and we can see that the points which represent our numerical data fit the sine line very well. We can see that when the value of $m^{2}$ increases, the maximum current $J_{\max }$ will grow. The dependence of $J_{\max }$ on the temperature $T$ is shown on the right panel of Fig. 2. The graph shows that $J_{\max } / T_{\mathrm{c}}^{2}$ increases with growing $T / T_{\mathrm{c}}$; however, in the s-N-s or p-N-p Josephson junction $[14,18], J_{\max } / T_{\mathrm{c}}^{2}$ decays with increasing $T / T_{\mathrm{c}}$. The reason is that in the s-wave and $\mathrm{p}$ wave coexisting region [28], the condensation of the s-wave decreases and the condensation of the p-wave increases with growing temperature, respectively. But the total condensation would decrease when the temperature drops. So, the $J_{\max }$ decreases with rising $T$.

The relationship of between $J_{\max } / T_{\mathrm{c}}^{2}$ and $\ell$ is shown on the left panel of Fig. 3. $J_{\max } / T_{\mathrm{c}}^{2}$ will decay with $\ell$ exponentially and the change will be larger when $m^{2}$ becomes larger. The total condensation in $y=0$ when the current $J=0$ is the sum of s-wave and p-wave condensation. Here, we define

$$
\langle\mathcal{O}(0)\rangle_{J=0}=\left\langle\mathcal{O}_{s}(0)\right\rangle_{J=0} / T_{\mathrm{c}}^{\Delta}+\left\langle\mathcal{O}_{p}(0)\right\rangle_{J=0} / T_{\mathrm{c}}^{2} .
$$

We plot the $\langle\mathcal{O}(0)\rangle_{J=0}$ on the right panel in Fig. 3. $\langle\mathcal{O}(0)\rangle_{J=0}$ also decays with growing $\ell$ exponentially and $\langle\mathcal{O}(0)\rangle_{J=0}$ becomes larger with increasing $m^{2}$. We have

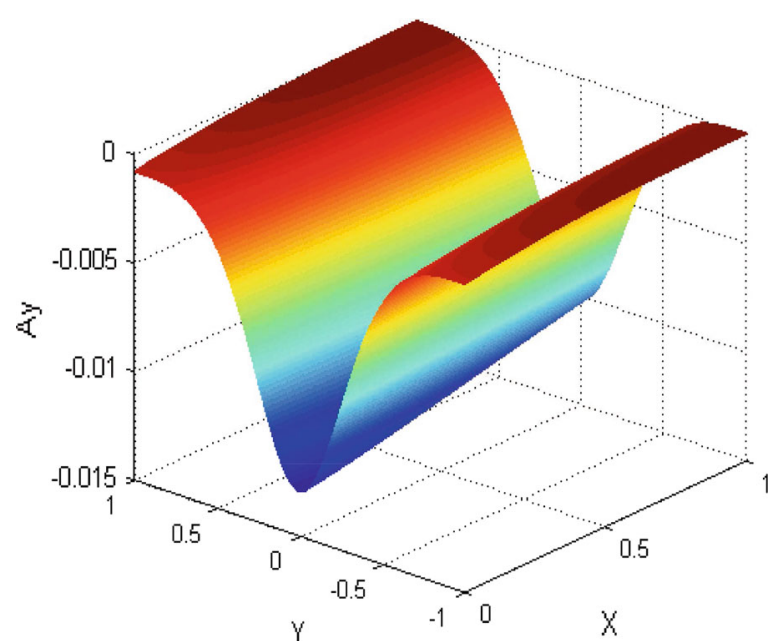

Fig. 1 We represent the components $\mu(y)$ and $A_{y}$ of Yang-Mills fields. Left The profile of $\mu(y)$. Right The figure of $A_{y}$. The parameters are $m^{2}=-33 / 16, \mu=7.6, \epsilon=0.25, \sigma=0.5, \ell=3$, and $\beta=1$. We can see that these two figures have symmetry 

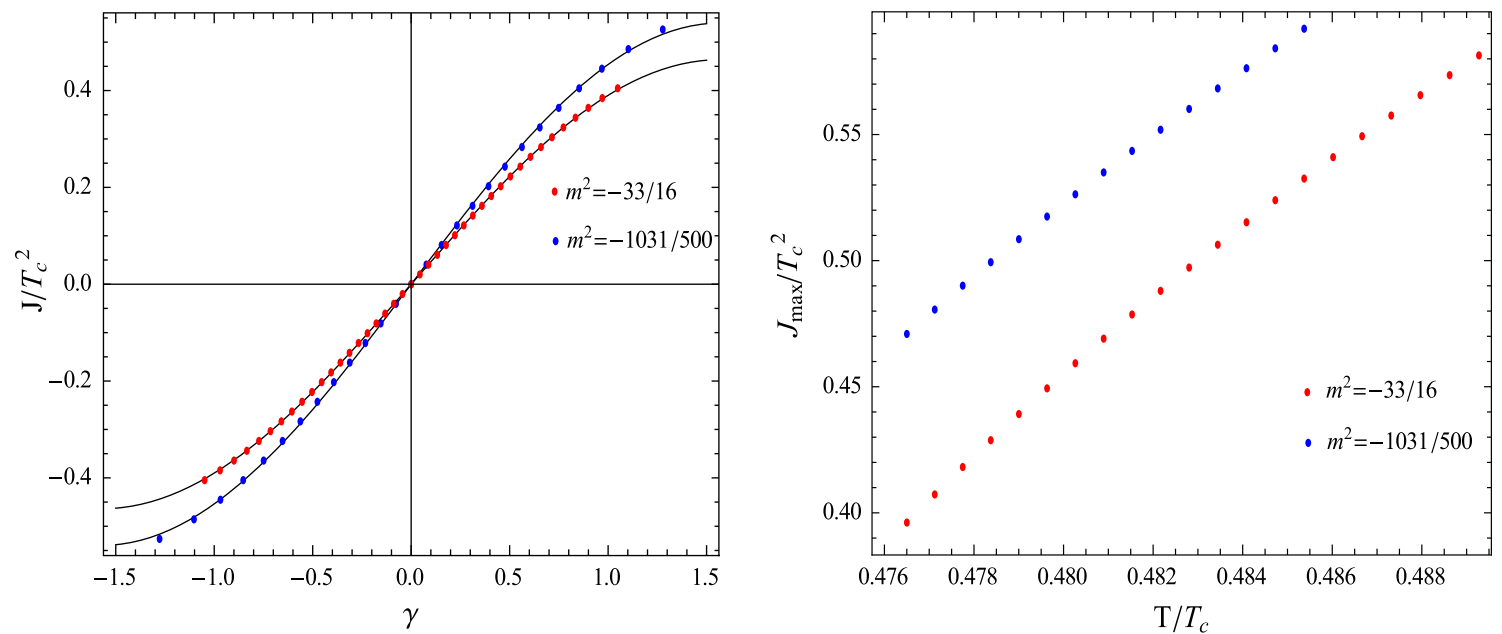

Fig. 2 Left The current $J / T_{\mathrm{c}}^{2}$ as a sine function of the difference phase $\gamma$. Right The $J_{\max } / T_{\mathrm{c}}^{2}$ increases with growing $T / T_{\mathrm{c}}$. The black lines are sine curves and the points with $m^{2}=-33 / 16$ (red), $-1031 / 500$ (blue) are numerical results. The parameters are $\mu=7.6, \epsilon=0.25, \sigma=0.5$, $\ell=3$, and $\beta=1$

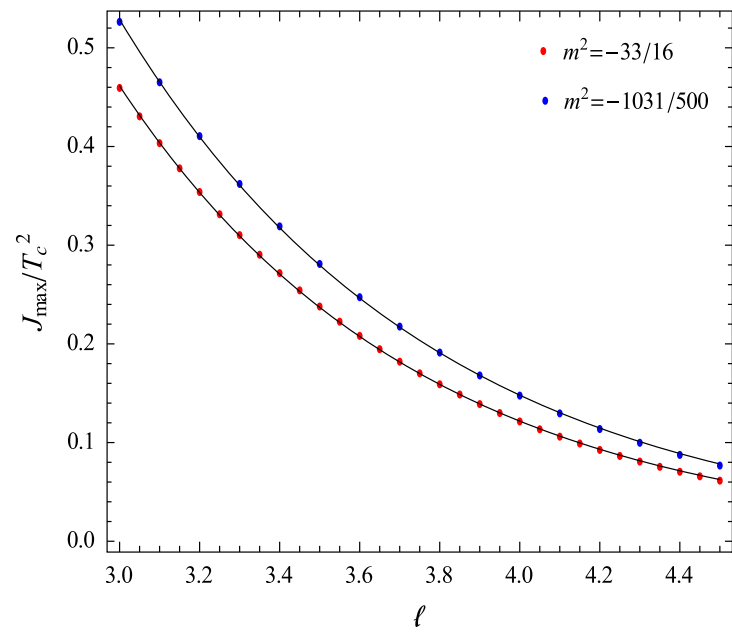

Fig. 3 Left The maximum current $J_{\max }$ as exponential function of $\ell$. Right The total condensate $\langle\mathcal{O}(0)\rangle_{J=0}$ as exponential function of $\ell$. Our numerical results are the points with $m^{2}=-33 / 16($ red $),-1031 / 500$

$$
\left\{\begin{array}{l}
J_{\max } / T_{\mathrm{c}}^{2} \approx 25.12 e^{-\ell / 0.7505} \\
\langle\mathcal{O}(0)\rangle_{J=0} \approx 22.03 e^{-\ell /(2 \times 0.7534)} .
\end{array} \text { for } m^{2}=-33 / 16,\right.
$$

We can obtain the coherence length $(0.7505,0.7534)$ from the above two equations, respectively. The error of the two values is about $0.4 \%$. We have

$$
\left\{\begin{array}{l}
J_{\max } / T_{\mathrm{c}}^{2} \approx 24.08 e^{-\ell / 0.7856} \\
\langle\mathcal{O}(0)\rangle_{J=0} \approx 20.66 e^{-\ell /(2 \times 0.8295)} .
\end{array} \quad \text { for } m^{2}=-1031 / 500,\right.
$$

The coherence length $(0.7856,0.8295)$ is obtained from the above two equations, respectively. The error of the two values is about $5.6 \%$.

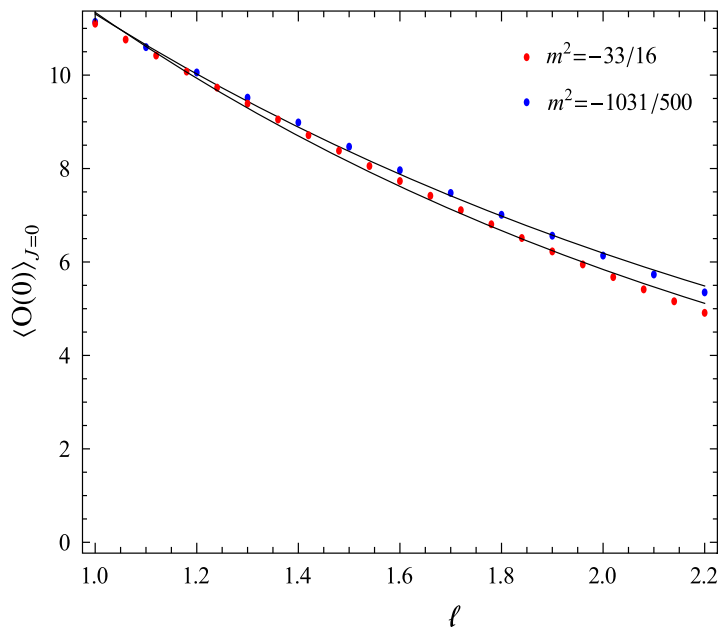

(blue). In all the plots, we use $\mu=7.6, \epsilon=0.25, \sigma=0.5$, and $\beta=1$. The numerical data fit the exponential curves well

\section{$3.2 \mathrm{~s}+\mathrm{p}-\mathrm{N}-\mathrm{s}$ Josephson junction}

In this subsection, the model of the s+p-N-s Josephson junction will be constructed. We tune the chemical potential $\mu(y)$ such that $\mu(-\infty)$ is in the $\mathrm{s}+\mathrm{p}$ coexisting region $\mu_{c 1} \sim \mu_{c 2}$ and $\mu(+\infty)>\mu_{c 2}$ is in the pure s-wave phase region. Because the superconductor condensations in the two leads are not symmetrical, the phase difference $\gamma$ can be calculated by $(2.21)$

$\gamma=-\int_{-\infty}^{0} \mathrm{~d} y[v(y)-v(-\infty)]-\int_{0}^{+\infty} \mathrm{d} y[v(y)-v(+\infty)]$. 

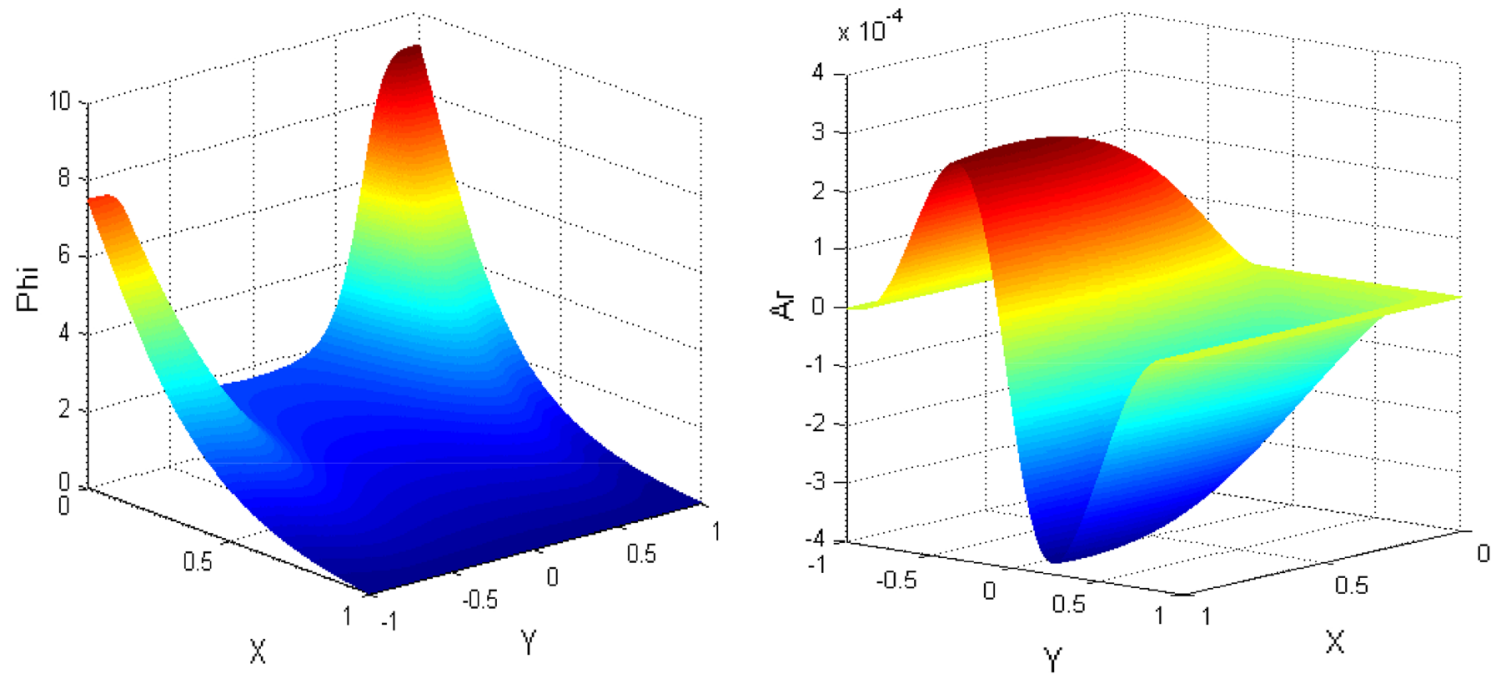

Fig. 4 We represent the components $\phi$ and $A_{r}$ of the Yang-Mills field. Left The profile of $\phi$. Right The figure of $A_{r}$. The parameters are $m^{2}=-33 / 16, \mu=8.3, \epsilon=0.3, \sigma=0.5, \ell=3$, and $\beta=1.286$. We can see that these two figures are not symmetrical
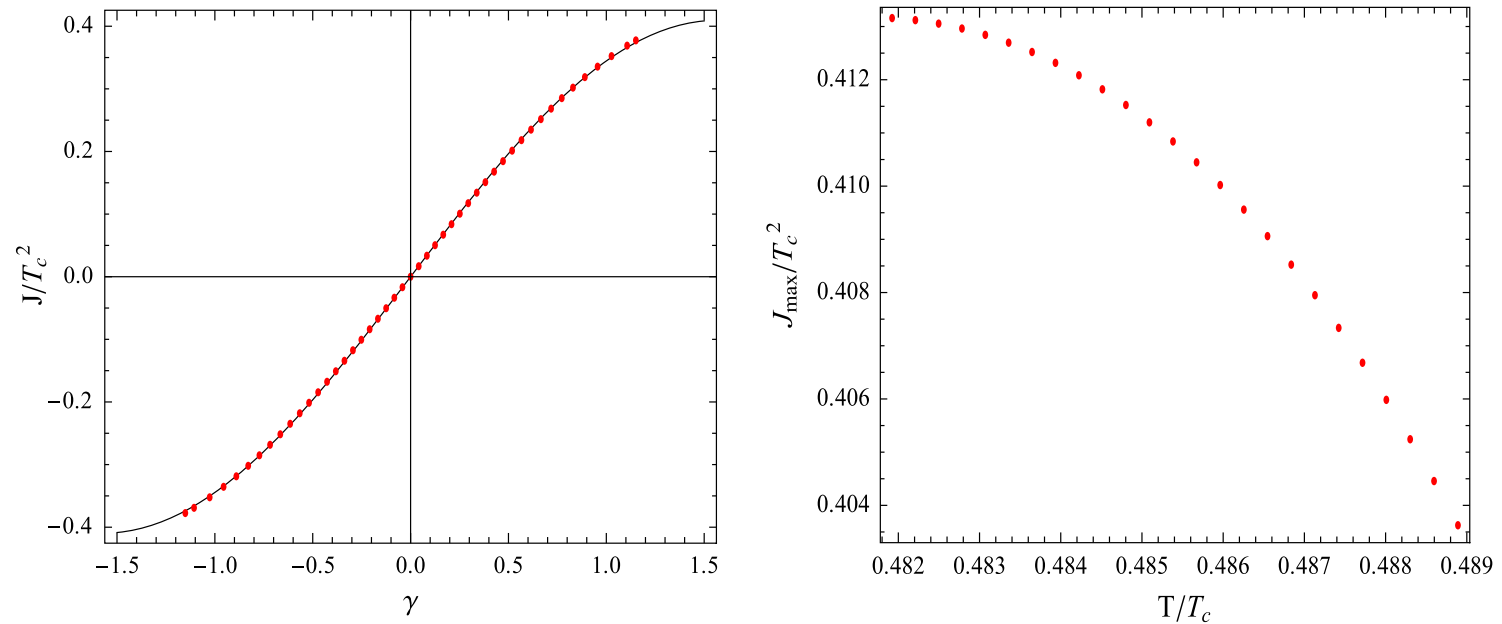

Fig. 5 Left The current $J / T_{\mathrm{c}}^{2}$ as sine function of phase difference $\gamma$. Right The $J_{\max } / T_{\mathrm{c}}^{2}$ decays with growing $T / T_{\mathrm{c}}$. The parameters are $m^{2}=-33 / 16, \mu=8.3, \epsilon=0.3, \sigma=0.5, \ell=3$, and $\beta=1.286$. The numerical data fit the exponential curves well

First, we show the profiles of $A_{t}^{1}$ and $A_{y}^{1}$ in Fig. 4, with the parameters $m^{2}=-33 / 16, \mu=8.3, \epsilon=0.3, \sigma=0.5$, $\ell=3$, and $\beta=1.286$.

The dependence of the current $J$ on the phase difference $\gamma$ across the junction is shown on the left panel of Fig. 5. The figure shows that $J$ is proportional to the sine of $\gamma$.

$J / T_{\mathrm{c}}^{2} \approx 0.4095 \sin \gamma$, for $m^{2}=-33 / 16$.

From the above result, we can see our numerical data which is drawn with the red points fits sine line very well. The dependence of $J_{\max }$ on the temperature $T$ is shown on the right panel of Fig. 5. The graph shows that $J_{\max } / T_{\mathrm{c}}^{2}$ decreases with growing $T / T_{\mathrm{c}}$.

The dependence of $J_{\max }$ on the width $\ell$ of the gap is shown on the left panel of Fig. 6 . The figure shows that $J_{\max } / T_{\mathrm{c}}^{2}$ decays with growing $\ell$ exponentially. The dependence of $\langle\mathcal{O}(0)\rangle_{J=0}$ on the width $\ell$ of the gap is shown on the right panel of Fig. 6. The figure predicts that $\langle\mathcal{O}(0)\rangle_{J=0}$ decays with growing $\ell$ exponentially. We have

$$
\left\{\begin{array}{l}
J_{\max } / T_{\mathrm{c}}^{2} \approx 27.07 e^{-\ell / 0.7138} \\
\langle\mathcal{O}(0)\rangle_{J=0} \approx 26.91 e^{-\ell /(2 \times 0.6567)} .
\end{array} \quad \text { for } m^{2}=-33 / 16,\right.
$$

The coherence length $(0.7138,0.6567)$ can be obtained from these equations. The error of these two values is about $8 \%$.

\section{3 s+p-N-p Josephson junction}

In this subsection, let us to continue to study the $\mathrm{s}+\mathrm{p}-\mathrm{N}$ p Josephson junction. We tune the chemical potential $\mu(y)$ such that $\mu(-\infty)$ is in the $\mathrm{s}+\mathrm{p}$ coexisting region $\mu_{c 1} \sim \mu_{c 2}$ 

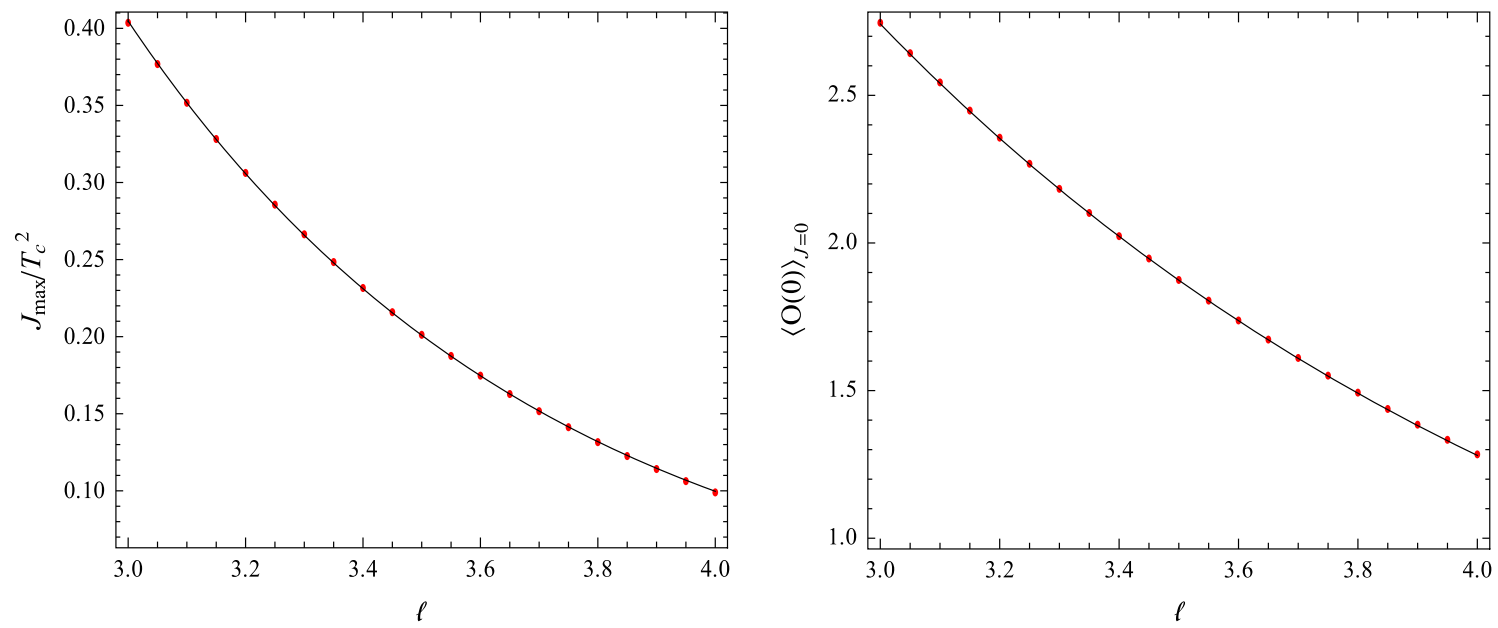

Fig. 6 Left The maximum current $J_{\max } / T_{\mathrm{c}}^{2}$ as an exponential function of the width $\ell$. Right The total condensate $\langle\mathcal{O}(0)\rangle_{J=0}$ as an exponential function of the width $\ell$. In all the plots, we use $m^{2}=-33 / 16, \mu=8.3, \epsilon=0.3, \sigma=0.5$, and $\beta=1.286$. The numerical data fit exponential curves well
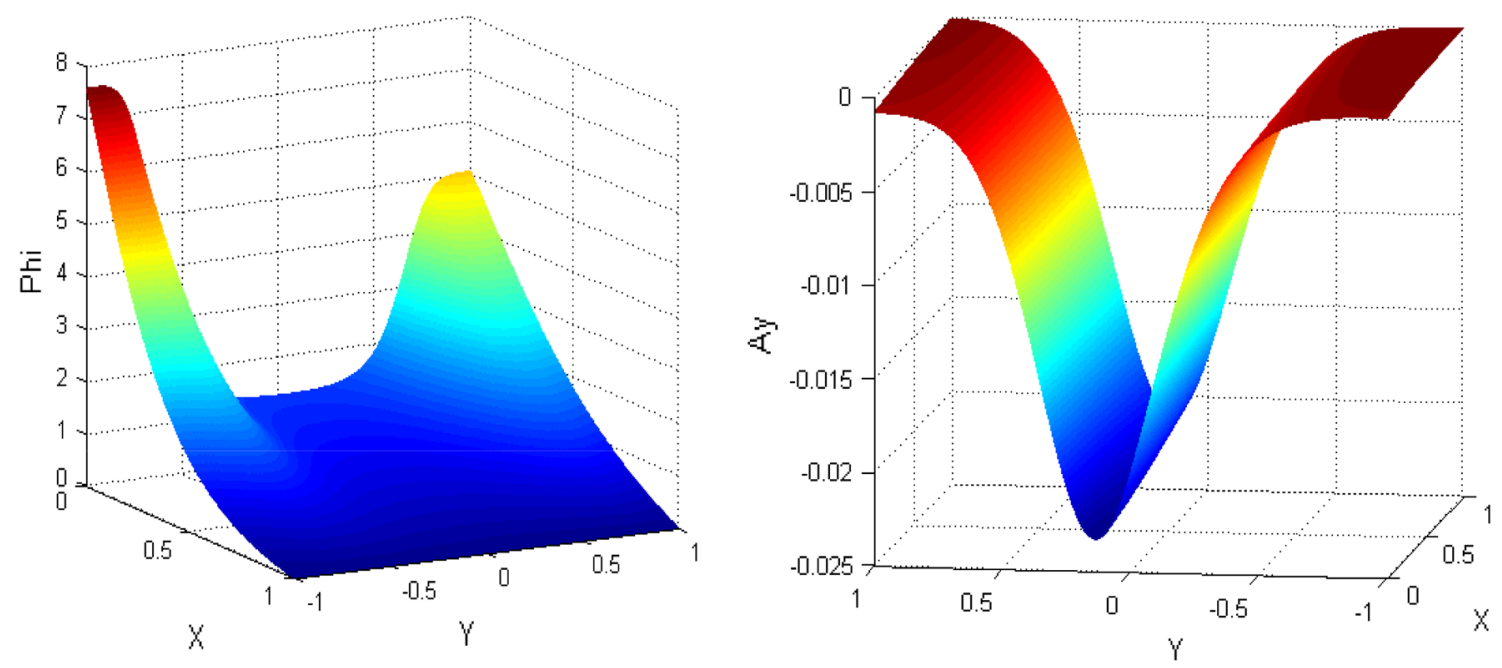

Fig. 7 We represent the components $\phi$ and $A_{y}$ of the Yang-Mills field. Left The profile of $\phi$. Right The figure of $A_{y}$. The parameters are $m^{2}=-33 / 16, \mu=6.3, \epsilon=0.0, \sigma=0.5, \ell=3$, and $\beta=0.6$. We can see that these two figures are not symmetrical

and $\mu(+\infty)<\mu_{c 1}$ is in the pure p-wave phase region. We still calculate the phase difference $\gamma$ from Eq. (3.3). The profiles of $A_{t}^{1}$ and $A_{y}^{1}$ are shown in Fig. 7, with the parameters $m^{2}=-33 / 16, \mu=6.3, \epsilon=0.0, \sigma=0.5, \ell=3$, and $\beta=0.6$.

The dots are determined by the DC current $J$ and the phase difference $\gamma$ across the junction is shown on the left panel of Fig. 8. With data fitting, we can see that $J$ is proportional to the sine of $\gamma$. We have

$J / T_{\mathrm{c}}^{2} \approx \begin{cases}0.4999 \sin \gamma & \text { for } m^{2}=-33 / 16 \\ 0.5318 \sin \gamma & \text { for } m^{2}=-1031 / 500 .\end{cases}$

From the figure, it is shown that when the value of $m^{2}$ increases the maximum current $J_{\max }$ will grow. The depen- dence of $J_{\max }$ on the temperature $T$ is shown on the right panel of Fig. 8. The graph shows that $J_{\max } / T_{\mathrm{c}}^{2}$ increases with growing $T / T_{\mathrm{c}}$, which is for the same reason as the case of the $s+p-N-s+p$ junction.

In the left panel of Fig. 3, the dependence of $J_{\max } / T_{\mathrm{c}}^{2}$ on $\ell$ is shown. We can see that $J_{\max } / T_{\mathrm{c}}^{2}$ decays with $\ell$ exponentially and the change will be larger when $m^{2}$ becomes larger. Furthermore, we show the dependence of $\langle\mathcal{O}(0)\rangle_{J=0}$ on $\ell$ in the right panel in Fig. 9. $\langle\mathcal{O}(0)\rangle_{J=0}$ also decays with growing $\ell$ exponentially and $\langle\mathcal{O}(0)\rangle_{J=0}$ becomes larger with increasing $m^{2}$. We have

$$
\left\{\begin{array}{l}
J_{\max } / T_{\mathrm{c}}^{2} \approx 7.843 e^{-\ell / 0.7376} \\
\langle\mathcal{O}(0)\rangle_{J=0} \approx 15.61 e^{-\ell /(2 \times 0.6021)} .
\end{array} \text { for } m^{2}=-33 / 16,\right.
$$



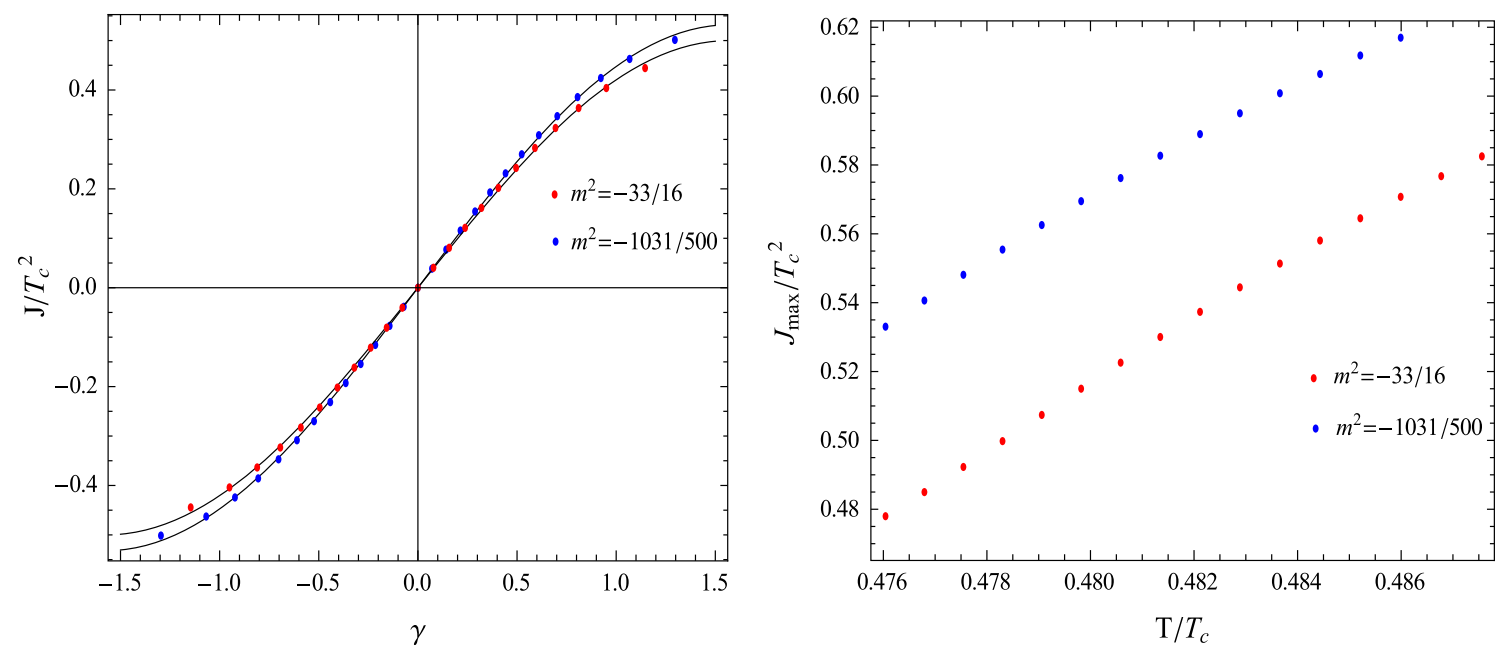

Fig. 8 Left The current $J / T_{\mathrm{c}}^{2}$ as a sine function of the difference phase $\gamma$. Right $J_{\max } / T_{\mathrm{c}}^{2}$ increases with growing $T / T_{\mathrm{c}}$. The black lines are sine curves and the points with $m^{2}=-33 / 16$ (red), $-1031 / 500$ (blue) are numerical results. The parameters are $\mu=6.3, \epsilon=0.0, \sigma=0.5, \ell=3$, and $\beta=0.6$.

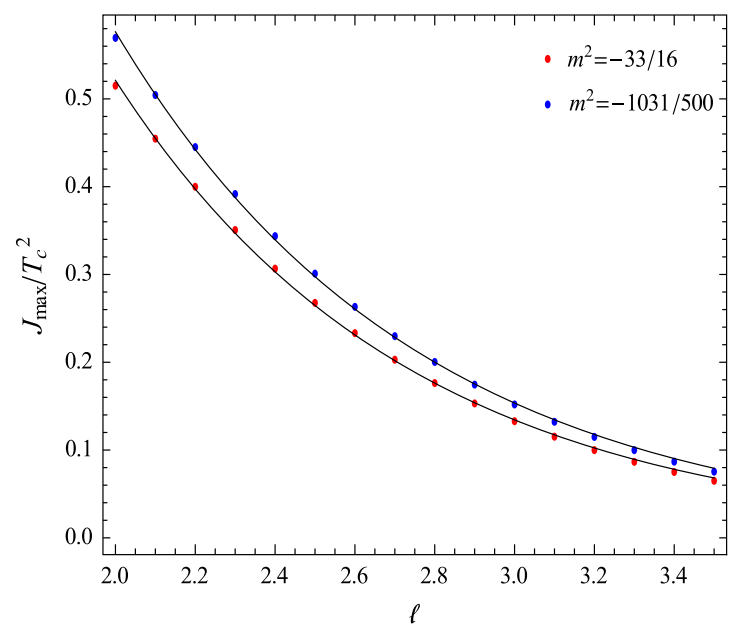

Fig. 9 Left The maximum current $J_{\max }$ as an exponential function of $\ell$. Right The total condensate $\langle\mathcal{O}(0)\rangle_{J=0}$ as an exponential function of $\ell$. Our numerical results are the points with $m^{2}=-33 / 16$ (red),

From the above result, we can obtain the coherence length $(0.7376,0.6021)$ from the above two equations, respectively. The error of the two values is about $18.4 \%$. We have

$\left\{\begin{array}{l}J_{\max } / T_{\mathrm{c}}^{2} \approx 8.129 e^{-\ell / 0.7558} \\ \langle\mathcal{O}(0)\rangle_{J=0} \approx 14.68 e^{-\ell /(2 \times 0.6337)} .\end{array} \quad\right.$ for $m^{2}=-1031 / 500$,

Similarly, the coherence length $(0.7558,0.6337)$ is obtained from the above two equations, respectively. The error of the two values is about $16.2 \%$.

\section{4 s-N-p Josephson junction}

In this subsection, we will construct the hybrid model of the s-wave and p-wave Josephson junction, namely, the s-

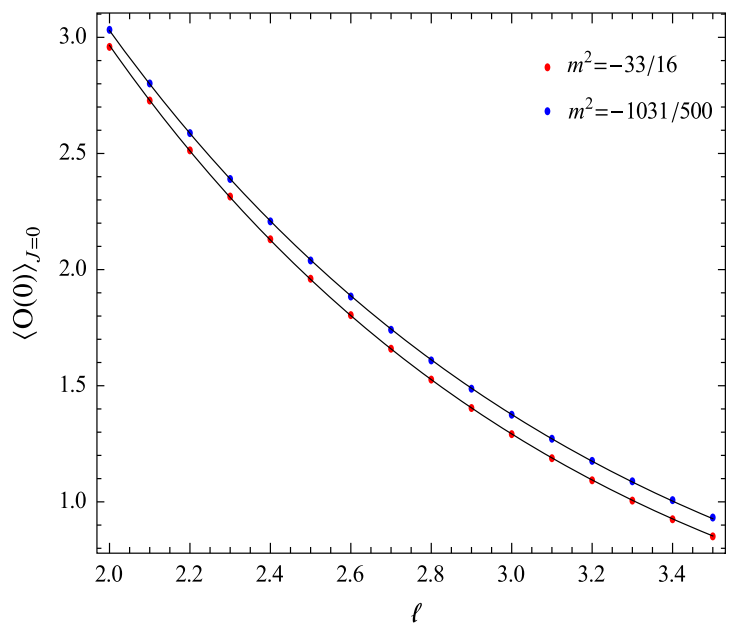

$-1031 / 500$ (blue). In all the plots, we use $\mu=6.3, \epsilon=0.0, \sigma=0.5$, and $\beta=0.6$. The numerical data fit the exponential curves well

N-p Josephson junction. We tune the value of the chemical potential $\mu(y)$ such that $\mu(-\infty)>\mu_{c 2}$ is in the pure swave region and $\mu(+\infty)<\mu_{c 1}$ is in the pure p-wave phase region. The phase difference $\gamma$ can be obtained in Eq. (3.3). The profiles of $A_{t}^{1}$ and $A_{y}^{1}$ are shown in Fig. 10, with the parameters $m^{2}=-33 / 16, \mu=7, \epsilon=0.0, \sigma=0.5, \ell=3$, and $\beta=0.6$.

We show the dependence of the current $J$ on the phase difference $\gamma$ across the junction on the left side of Fig. 11, in which it is shown that $J$ is proportional to the sine of $\gamma$. We have

$J / T_{\mathrm{c}}^{2} \approx 0.1166 \sin \gamma$, for $m^{2}=-33 / 16$ 

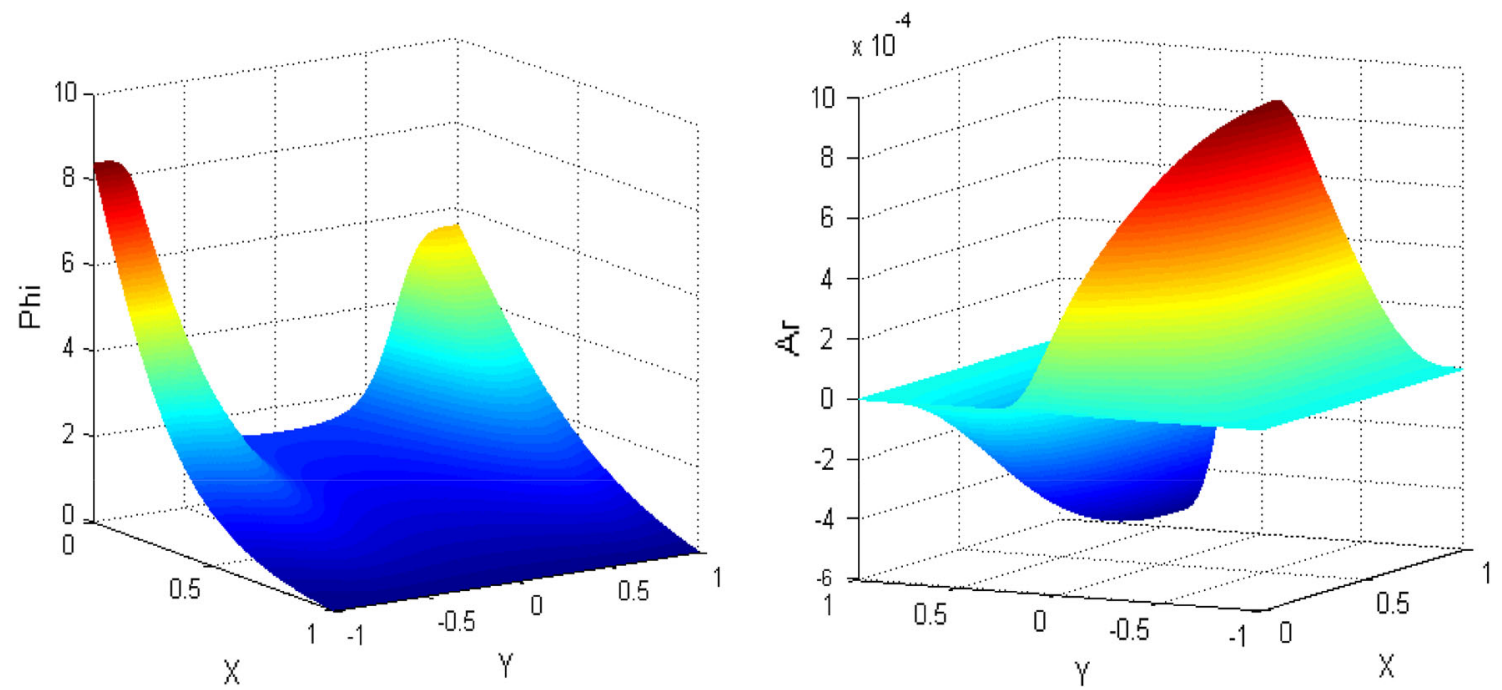

Fig. 10 We represent the components $\phi$ and $A_{y}$ of the Yang-Mills field. (Left) The profile of $\phi$. (Right) The figure of $A_{y}$. The parameters are $m^{2}=-33 / 16, \mu=7, \epsilon=0.0, \sigma=0.5, \ell=3$, and $\beta=0.6$. We can see that these two figures are not symmetrical
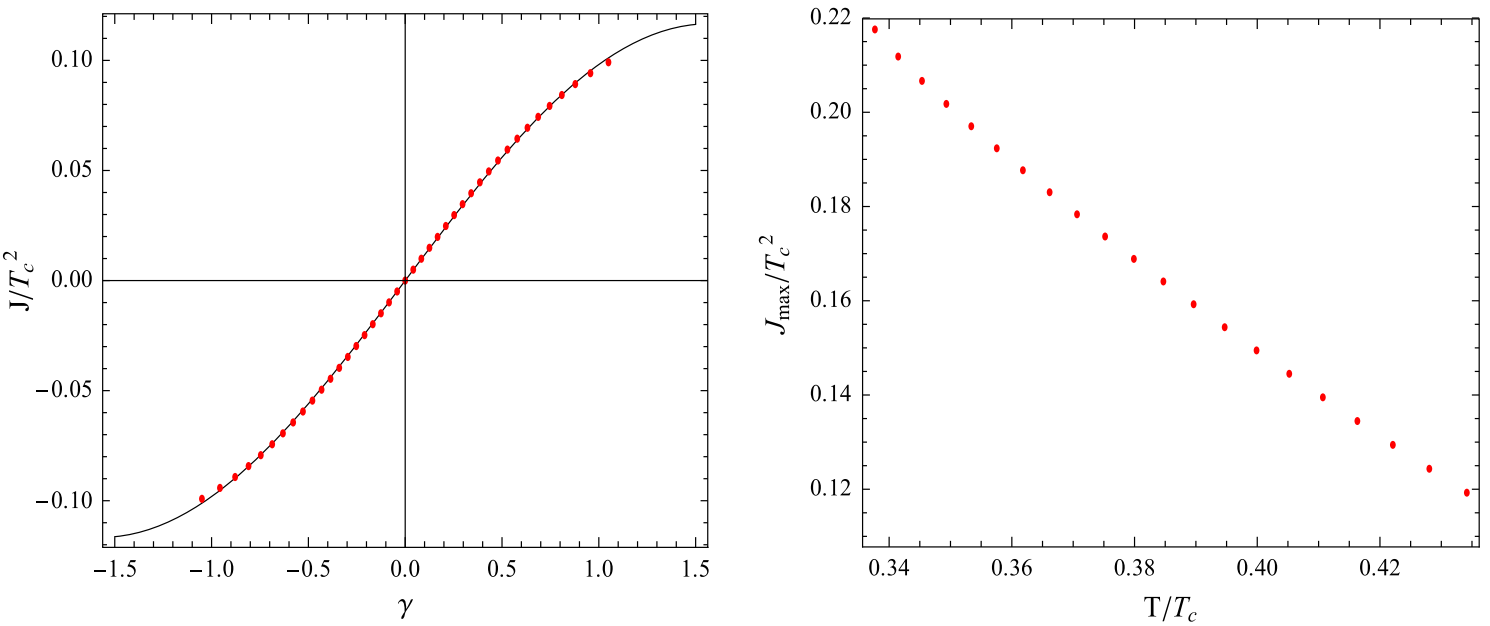

Fig. 11 Left The current $J / T_{\mathrm{c}}^{2}$ as a sine function of $\gamma$. Right $J_{\max } / T_{\mathrm{c}}^{2}$ decays with growing $T / T_{\mathrm{c}}$. In all the plots, we use $m^{2}=-33 / 16, \mu=7$, $\epsilon=0.0, \sigma=0.5, \ell=3$, and $\beta=0.6$. The numerical data fit the exponential curves well

The dependence of $J_{\max }$ on the temperature $T$ is shown on the right panel of Fig. 11, which shows that $J_{\max } / T_{\mathrm{c}}^{2}$ decreases with growing $T / T_{\mathrm{c}}$.

The dependence of $J_{\max }$ on the width $\ell$ of the gap is shown on the left panel of Fig. 12, which shows that $J_{\max } / T_{\mathrm{c}}^{2}$ decays with growing $\ell$ exponentially. The dependence of $\langle\mathcal{O}(0)\rangle_{J=0}$ on the width $\ell$ of the gap is shown on the right panel of Fig. 12 , which predicts that $\langle\mathcal{O}(0)\rangle_{J=0}$ decays with growing $\ell$ exponentially. We have

$$
\left\{\begin{array}{l}
J_{\max } / T_{\mathrm{c}}^{2} \approx 9.842 e^{-\ell / 0.6798} \\
\langle\mathcal{O}(0)\rangle_{J=0} \approx 12.95 e^{-\ell /(2 \times 0.6362)} .
\end{array} \text { for } m^{2}=-33 / 16\right.
$$

The coherence length $(0.6798,0.6362)$ can be obtained from these equations. The error of these two values is about $6.4 \%$.

\section{Conclusion and discussion}

In this paper, we set up a holographic model for a hybrid swave and p-wave DC Josephson junction with a scalar triplet charged under the $S U$ (2) gauge field in the background of a $(3+1)$-dimensional AdS black hole. We get a set of partial differential equations of fields that are nonlinear and coupled and solve them numerically. We construct a new chemical 

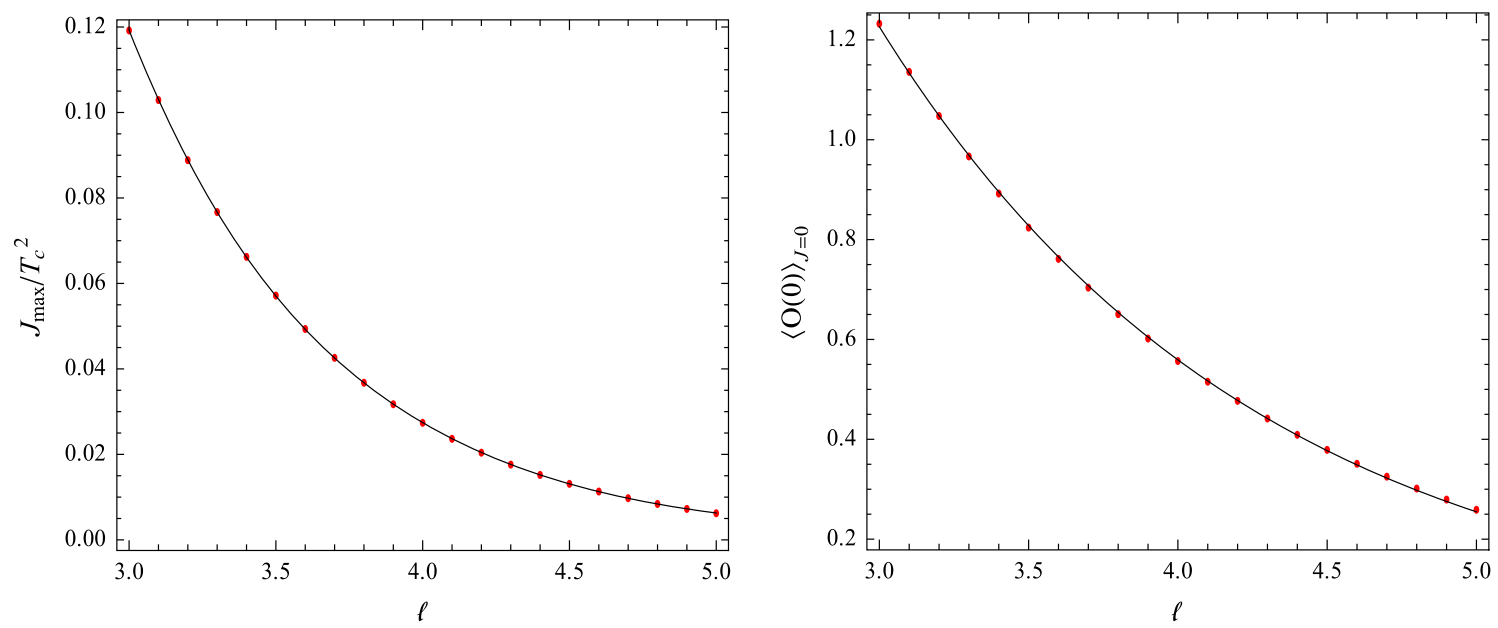

Fig. 12 Left The maximum current $J_{\max } / T_{\mathrm{c}}^{2}$ as an exponential function of $\ell$. Right The total condensate $\langle\mathcal{O}(0)\rangle_{J=0}$ as exponential function of $\ell$. In all the plots, we use $m^{2}=-33 / 16, \mu=7, \epsilon=0.0, \sigma=0.5$, and $\beta=0.6$. The numerical data fit the exponential curves well

potential $\mu(y)$ and tune the parameters in it, so the $\mathrm{s}+\mathrm{p}-\mathrm{N}-$ $\mathrm{s}+\mathrm{p}$ junction, $\mathrm{s}+\mathrm{p}-\mathrm{N}-\mathrm{s}$ junction, $\mathrm{s}+\mathrm{p}-\mathrm{N}-\mathrm{p}$ junction, and $\mathrm{s}-\mathrm{N}-\mathrm{p}$ junction can be obtained, respectively. For the four kinds of junctions, we find that the DC is proportional to the sine of the phase difference across the junction and the coherence lengths are different. We also study the relationship between $J_{\max } / T_{\mathrm{c}}^{2}$ and $\ell$, the total condensation $\langle\mathcal{O}(0)\rangle_{J=0}$ and $\ell, J_{\max } / T_{\mathrm{c}}^{2}$ and $T / T_{\mathrm{c}}$, respectively.

The reason we take $m^{2}=-33 / 16$ is that when $m^{2}<$ $-33 / 16$, the region of $s+p$ coexistence is too small, when $m^{2}>-33 / 16$, the value in the region of $\mathrm{s}+\mathrm{p}$ coexistence is too large for the junction, the numerical results are not good. It is well known that the Josephson period is $2 \pi$ in the $p_{y}$ wave $s-\mathrm{N}-\mathrm{p}$ junction, so our ansatz just corresponds to the $p_{y}$ wave junction. To our surprise, the periods of the currents are also $2 \pi$ in the remaining three kinds of junctions. For the $\mathrm{s}+\mathrm{p}-\mathrm{N}-\mathrm{s}+\mathrm{p}$ junction and the $\mathrm{s}+\mathrm{p}-\mathrm{N}-\mathrm{p}$ junction, we take different values of $m^{2}=-33 / 16,-1031 / 500$ and find that when the value becomes larger, $J, J_{\max } / T_{\mathrm{c}}^{2}$, and $\langle\mathcal{O}(0)\rangle_{J=0}$ will become larger. The reason we take another value of $m^{2}=-1031 / 500$ is that the region of $\mathrm{s}+\mathrm{p}$ coexistence is small, we should take the other value of $m^{2}$ as it approaches the $m^{2}=-33 / 16$. The maximum current increases with the growing temperature in the $\mathrm{s}+\mathrm{p}-\mathrm{N}-\mathrm{s}+\mathrm{p}$ and the $\mathrm{s}+\mathrm{p}-\mathrm{N}-\mathrm{p}$ junction. Except the $\mathrm{s}+\mathrm{p}-\mathrm{N}-\mathrm{s}+\mathrm{p}$ junction, the phase difference should be obtained by $\gamma=-\int_{-\infty}^{c} d y[v(y)-v(-\infty)]-$ $\int_{\mathrm{c}}^{+\infty} d y[v(y)-v(+\infty)]$ without loss of generality. When $c \neq 0$, the relationship between the current and the phase difference is $J / T_{\mathrm{c}}^{2}=\left(J_{\max } / T_{\mathrm{c}}^{2}\right) \sin (\gamma+\phi)$, where $\phi$ is the origin's phase difference and $\phi \neq 0$. So it is more convenient to set $c=0$ to make $\phi=0$.

Note that our model also can describe the s-wave superconductor, the $\mathrm{p}$-wave superconductor, the $\mathrm{s}+\mathrm{p}$ coexistence superconductor, the s-wave junction, and the p-wave junction. The $\mathrm{p}$-wave contains a $p_{x}$ wave and a $p_{y}$ wave, the
Josephson periods are $\pi$ and $2 \pi$, respectively. In the present study, this ansatz just describes the $p_{y}$ wave. So, it should be of great interest to construct an ansatz which can describe a $p_{x}$ wave and a $p_{y}$ wave, respectively.

Finally, we have studied the hybrid and coexisting s-wave and p-wave junctions with the probe limit, and we would like to study these kinds of junctions with the gravity backreaction in the future. So far, we have studied the hybrid and coexisting s-wave and p-wave DC Josephson junctions in the AdS black hole background. It is intended to study these DC junctions by taking an AdS soliton as the geometry background in our further work.

Acknowledgments YQW would like to thank Li Li and Hai-Qing Zhang for very helpful discussion. SL and YQW were supported by the National Natural Science Foundation of China.

Open Access This article is distributed under the terms of the Creative Commons Attribution 4.0 International License (http://creativecomm ons.org/licenses/by/4.0/), which permits unrestricted use, distribution, and reproduction in any medium, provided you give appropriate credit to the original author(s) and the source, provide a link to the Creative Commons license, and indicate if changes were made.

Funded by SCOAP . $^{3}$

\section{References}

1. J.M. Maldacena, The large $\mathrm{N}$ limit of superconformal field theories and supergravity. Int. J. Theor. Phys. 38, 1113 (1999)

2. J.M. Maldacena, Adv. Theor. Math. Phys. 2, 231 (1998) arXiv:hep-th/9711200

3. S.S. Gubser, Breaking an Abelian gauge symmetry near a black hole horizon. Phys. Rev. D 78, 065034 (2008). arXiv:0801.2977 [hep-th]

4. S.A. Hartnoll, C.P. Herzog, G.T. Horowitz, Building a holographic superconductor. Phys. Rev. Lett. 101, 031601 (2008). arXiv:0803.3295 [hep-th]

5. S.A. Hartnoll, C.P. Herzog, G.T. Horowitz, Holographic superconductors. JHEP 0812, 015 (2008). arXiv:0810.1563 [hep-th] 
6. S.S. Gubser, S.S. Pufu, JHEP 0811, 033 (2008). arXiv:0805.2960 [hep-th]

7. J.W. Chen, Y.J. Kao, D. Maity, W.Y. Wen, C.P. Yeh, Phys. Rev. D 81, 106008 (2010). arXiv:1003.2991 [hep-th]

8. F. Benini, C.P. Herzog, R. Rahman, A. Yarom, JHEP 1011, 137 (2010). arXiv:1007.1981 [hep-th]

9. S.A. Hartnoll, Lectures on holographic methods for condensed matter physics. Class. Quant. Grav. 26, 224002 (2009). arXiv:0903.3246 [hep-th]

10. C.P. Herzog, Lectures on holographic superfluidity and superconductivity. J. Phys. A 42, 343001 (2009). arXiv:0904.1975 [hep-th]

11. G.T. Horowitz, Introduction to holographic superconductors. Lect. Notes Phys. 828, 313 (2011). arXiv:1002.1722 [hep-th]

12. R.G. Cai, L. Li, L.F. Li, R.Q. Yang, Sci. China Phys. Mech. Astron. 58, 060401 (2015). arXiv:1502.00437 [hep-th]

13. B.D. Josephson, Possible new effects in superconductive tunnelling. Phys. Lett. 1, 251 (1962)

14. G.T. Horowitz, J.E. Santos, B. Way, A holographic Josephson junction. Phys. Rev. Lett. 106, 221601 (2011). arXiv:1101.3326 [hepth]

15. Y.Q. Wang, Y.X. Liu, Z.H. Zhao, Holographic Josephson junction in $3+1$ dimensions. arXiv: 1104.4303 [hep-th]

16. M. Siani, On inhomogeneous holographic superconductors. arXiv: 1104.4463 [hep-th]

17. E. Kiritsis, V. Niarchos, Josephson junctions and AdS/CFT networks. JHEP 1107, 112 (2011). arXiv:1105.6100 [hep-th]. (Erratum-ibid. 1110, 095 (2011))

18. Y.Q. Wang, Y.X. Liu, Z.H. Zhao, Holographic p-wave Josephson junction. arXiv:1109.4426 [hep-th]

19. Y.Q. Wang, Y.X. Liu, R.G. Cai, S. Takeuchi, H.Q. Zhang, Holographic SIS Josephson junction. JHEP 1209, 058 (2012). arXiv:1205.4406 [hep-th]

20. R.G. Cai, Y.Q. Wang, H.Q. Zhang, JHEP 1401, 039 (2014). arXiv: 1308.5088 [hep-th]

21. S. Takeuchi, Int. J. Mod. Phys. A 30(09), 1550040 (2015). arXiv:1309.5641 [hep-th]

22. H.F. Li, L. Li, Y.Q. Wang, H.Q. Zhang, JHEP 1412, 099 (2014). arXiv: 1410.5578 [hep-th]

23. P. Basu, J. He, A. Mukherjee, M. Rozali, H.H. Shieh, Competing holographic orders. JHEP 1010, 092 (2010). arXiv: 1007.3480 [hep-th]

24. D. Musso, JHEP 1306, 083 (2013). arXiv:1302.7205 [hep-th]
25. R.G. Cai, L. Li, L.F. Li, Y.Q. Wang, Competition and coexistence of order parameters in holographic multi-band superconductors. JHEP 1309, 074 (2013). arXiv:1307.2768 [hep-th]

26. A. Amoretti, A. Braggio, N. Maggiore, N. Magnoli, D. Musso, JHEP 1401, 054 (2014). arXiv:1309.5093 [hep-th]

27. L.F. Li, R.G. Cai, L. Li, Y.Q. Wang, Competition between s-wave order and d-wave order in holographic superconductors. JHEP 1408, 164 (2014). arXiv:1405.0382 [hep-th]

28. Z.Y. Nie, R.G. Cai, X. Gao, H. Zeng, Competition between the swave and p-wave superconductivity phases in a holographic model. JHEP 1311, 087 (2013). arXiv:1309.2204 [hep-th]

29. I. Amado, D. Arean, A. Jimenez-Alba, L. Melgar, I. Salazar Landea, Phys. Rev. D 89(2), 026009 (2014). arXiv:1309.5086 [hep-th]

30. Z.Y. Nie, R.G. Cai, X. Gao, L. Li, H. Zeng. arXiv:1501.00004 [hep-th]

31. P. Breitenlohner, D.Z. Freedman, Positive energy in anti-De Sitter backgrounds and gauged extended supergravity. Phys. Lett. B 115, 197 (1982)

32. P. Basu, A. Mukherjee, H.-H. Shieh, Supercurrent: vector hair for an AdS black hole. Phys. Rev. D 79, 045010 (2009). arXiv:0809.4494 [hep-th]

33. C.P. Herzog, P.K. Kovtun, D.T. Son, Holographic model of superfluidity. Phys. Rev. D 79, 066002 (2009). arXiv:0809.4870 [hep-th]

34. D. Arean, M. Bertolini, J. Evslin, T. Prochazka, On holographic superconductors with DC current. JHEP 1007, 060 (2010). arXiv:1003.5661 [hep-th]

35. J. Sonner, B. Withers, A gravity derivation of the TiszaLandau model in AdS/CFT. Phys. Rev. D 82, 026001 (2010). arXiv: 1004.2707 [hep-th]

36. G.T. Horowitz, M.M. Roberts, Holographic superconductors with various condensates. Phys. Rev. D 78, 126008 (2008). arXiv:0810.1077 [hep-th]

37. D. Arean, P. Basu, C. Krishnan, The many phases of holographic superfluids. JHEP 1010, 006 (2010). arXiv:1006.5165 [hep-th]

38. H.B. Zeng, W.M. Sun, H.S. Zong, Supercurrent in p-wave holographic superconductor. Phys. Rev. D 83, 046010 (2011). arXiv: 1010.5039 [hep-th]

39. D. Arean, M. Bertolini, C. Krishnan, T. Prochazka, JHEP 1103, 008 (2011). arXiv:1010.5777 [hep-th] 